\title{
Plant Immunity May Benefit Human Medicine
}

\author{
Ephraim Philip Lansky* and Eviatar Nevo
}

Institute of Evolution, Haifa University, Haifa, Israel

\begin{abstract}
Brachypodium distachyon, a model genomic organism, has also been studied metabolomically for its reactions to specific stimuli such as fungal infection or proximity to other botanical organisms. Coincidentally, the metabolic output in response to pathogenic fungi produces compounds (phospholipids) valuable in treating invasive fungal infections in humans. This leads to general hypothesis that specific stressing of plants may produce compounds of value for the treatment resulting from homologous or identical stressors, and so constitute a novel putative approach for pharmaceutical discovery and development. Some specific examples and suggestions for future study are considered.
\end{abstract}

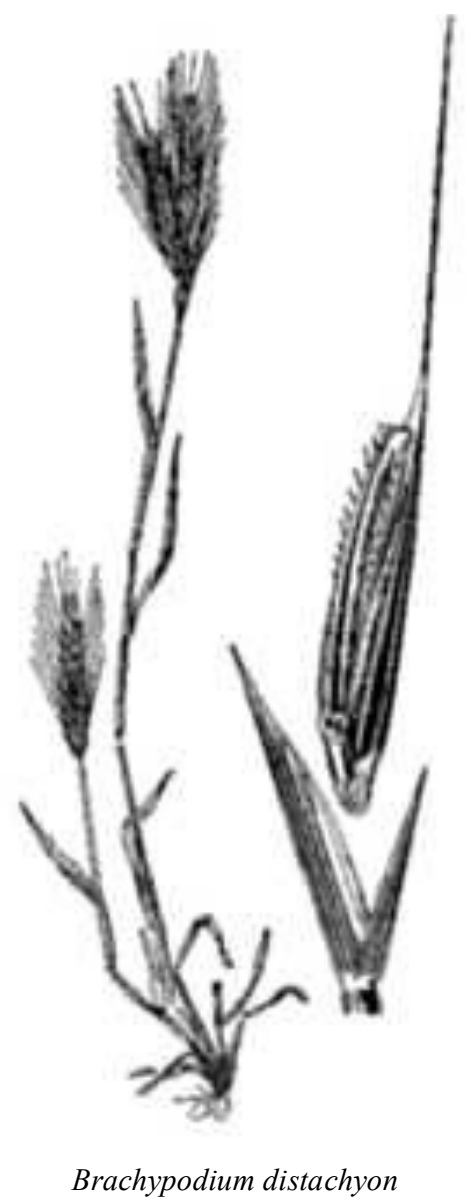

Fig. (1). Image from USDA-NRCS PLANTS Database / Hitchcock, A.S. (rev. A. Chase). 1950. Manual of the grasses of the United States. USDA Misc. Publ. No. 200. Washington, DC.

The diminutive grass, Brachypodium distachyon (Fig. 1), as its Greek name (Brachypodium = short foot) suggests, has a small phenotypic footprint with a correspondingly small genome of $300 \mathrm{Mb}$. Closely related to wheat, whose genome

*Address correspondence to this author at the Institute of Evolution, Haifa University, Haifa, Israel; Tel: +972 545273 156;

E-mail: elansky@research.haifa.ac.il size is 16-700 Mb, Brachypodium has become a favored model for understanding grass biology and its relevance for improving the human food supply $[1,2]$.

Metabolomics combines bioinformatics and automated analytical chemistry to reveal diversity beyond the genome, transcriptome and proteome, specifically, to the totality of numerous metabolites, the metabolome. In one case, infrared fingerprinting of the metabolic reactions of the monocotyledon Brachypodium distachyon to physical proximity of the dicotyledon Arabidopsis thaliana was characterized [3]. Then, exposure of Brachypodium to the pathogenic cereal fungus, Magnaporthe grisea, the cause of rice blast disease, an item of biological warfare and a terrorist threat, was shown to produce a distinctive immunological response of downregulating phosphatidyl glycerol phospholipids and upregulating phosphatidic acid phospholipids, mediating disease resistance [4] through immunological defense mechanisms [5].

Curiously, these same phospholipids may both augment and modulate anti-fungal drugs in treating invasive fungal infection in humans. Thus, when the anti-fungal agent amphotericin B is sequestered in phospholipid liposomes, it produces less accompanying inflammation, potentially lifesaving when immunity is crippled following long-standing corticosteroid usage [6]. And, empty liposomes consisting entirely of phospholipids produce anti-fungal effect comparable or even superior to amophotericin-B alone [7]!

Phospholipids may help prevent Alzheimer's or Parkinson's disease [8], function as antibiotics [9, 10], control photosynthesis of plants [11], and help treating respiratory distress syndromes [12] and autoimmune disorders [13]. Their effects against fungi like Magnaporthe grisea may provide Homo sapiens, as well as Brachypodium distachyon, a competitive edge for survival.

The soil phytopathogen, Agrobacterium tumefaciens, transforming plant cells to produce the tumor-like "crown gall disease," also experimentally transforms normal human cells to neoplastic ones [14]. So, could Agrobacterium tumefaciens be employed to elicit anti-tumor chemicals by plants that might also prove of value for human oncology? 
Oxidative tension in both plants and animals triggers compensatory reactions. In animals, inflammation or angiogenesis may be the result. Hydrogen peroxide, a source of free radicals and instigator of oxidative tension, stimulates melons to produce sugar [15]. Metabolomics could reveal other compounds melons produce in response to hydrogen peroxide besides sugar, and these might be relevant as animal antioxidants or signal response modifiers to otherwise mollify or negate oxidative damage.

Sucrose loading in plants reveals the effects complementary to those of stress and copper on RNA and proteins [16]. Metabolomics could be expected to reveal additional detail at the level of small molecule metabolites. Could copper stress to a plant produce compounds valuable in the treatment of Wilson's disease, a disorder of copper metabolism, in humans? Did water excess to the plants produce factors useful in treating mammalian diabetes insipidus? Could plant salt-stress yield agents for human renal disease to correct hypernatremia, hyponatremia or other mineral abnormalities? Overcrowding of rodents in cages produces the corticosteroid neurological stress responses known as "experimental neurosis." Could such psychoneurological abonormalities be ameliorated by compounds produced by plants as immunological reactions to homologous conditions of crowding?

In that plants and animals share numerous biochemical pathways that have evolved from common mechanisms and genetic roots, such questions deserve to be asked. Brachypodium distachyon, celebrated by scientists worldwide as a model genomic organism, may also prove itself as a model for metabolomics. Short genes likely beget relatively small metabolomes, and the absence of any marked drug effects from this simple grass makes it ideal for use in experiments to test teasing of plants to produce immunnologically customized, secondary metabolites as putative therapy for specific and varied human medical problems.

The secondary metabolites that plants produce for their own purposes have underlined herbal and nutritional treatments for millenia. Many of these compounds, most notably resveratrol, are recognized as "phytoalexins" and have been and are the subject of ongoing "translational research" and clinical investigations $[17,18]$. New is the use of computing power sufficient for revealing the complexity of the plant's chemical signatures under particular environmental assaults. With the derivative metabolomics, it may even now be possible to expose previously unappreciated inter-kingdom metabolic networks connecting the stress-specific immunochemical output of plants to human biochemical byways for containing disease and promoting health.

\section{REFERENCES}

[1] Draper J, Mur LA, Jenkins G, et al. Brachypodium distachyon. A new model system for functional genomics in grasses. Plant Physiol 2001; 127: 1539-55.

[2] Opanowicz M, Vain P, Draper J, et al. Brachypodium distachyon: making hay with a wild grass. Trends Plant Sci 2008; 13: 172-7.

[3] Gidman E, Goodacre R, Emmett B, et al. Investigating plant-plant interference by metabolic fingerprinting. Phytochemistry 2003; 63: 705-10.

[4] Allwood JW, Ellis DI, Heald JK, et al. Metabolomic approaches reveal that phosphatidic and phosphatidyl glycerol phospholipids are major discriminatory non-polar metabolites in responses by Brachypodium distachyon to challenge by magnaporthe grisea. Plant J 2006; 46: 351-68.

[5] Jones JD, Dangl JL. The plant immune system. Nature 2006; 444 : 323-9.

[6] Ben-Ami R, Lewis RE, Kontoyiannis DP. Immunocompromised hosts: immunopharmacology of modern antifungals. Clin Infect Dis 2008; 47: 226-35.

[7] Lewis RE, Chamilos G, Prince RA, Kontoyiannis DP. Pretreatment with empty liposomes attenuates the immunopathology of invasive pulmonary aspergillosis in corticosteroid-immunosuppressed mice. Antimicrob Agents Chemother 2007; 51: 1078-81.

[8] Pope S, Land JM, Heales SJ. Oxidative stress and mitochondrial dysfunction in neurodegeneration; cardiolipin a critical target? Biochim Biophys Acta 2008; 1777: 794-9.

[9] Schlame M. Cardiolipin synthesis for the assembly of bacterial and mitochondrial membranes. J Lipid Res 2008; 49: 1607-20.

[10] Mileykovskaya E. Subcellular localization of Escherichia coli osmosensory transporter ProP: focus on cardiolipin membrane domains. Mol Microbiol 2007; 64: 1419-22.

[11] Wada H, Murata N. The essential role of phosphatidylglycerol in photosynthesis. Photosynth Res 2007; 92: 205-15.

[12] Donn SM, Sinha SK. Aerosolized lucinactant: a potential alternative to intratracheal surfactant replacement therapy. Expert Opin Pharmacother 2008; 9: 475-8.

[13] Lockshin MD. Update on antiphospholipid syndrome. Bull NYU Hosp Jt Dis 2008; 66(3): 195-7.

[14] Kunik T, Tzfira T, Kapulnik Y, et al. Genetic transformation of HeLa cells by Agrobacterium. Proc Natl Acad Sci USA 2001; 98 : 1871-6.

[15] Ozaki K, Uchida A, Takabe T, et al. Enrichment of sugar content in melon fruits by hydrogen peroxide treatment. J Plant Physiol 2009; 166(6): 569-78.

[16] Dugas DV, Bartel B. Sucrose induction of Arabidopsis miR398 represses two $\mathrm{Cu} / \mathrm{Zn}$ superoxide dismutases. Plant Mol Biol 2008; 67: 403-17.

[17] Cucciolla V, Borriello A, Oliva A, et al. Resveratrol: from basic science to the clinic. Cell Cycle 2007; 6: 2495-510.

[18] Maor R, Shirasu K. The arms race continues: battle strategies between plants and fungal pathogens. Curr Opin Microbiol 2005; 8: 399-404. 